\title{
Avaliação do controle postural e sua relação com o hemisfério acometido no Acidente Vascular Cerebral
}

Postural control evaluation and

its relationship with the impaired

FisiSenectus . Unochapecó Ano 6, n. 1 - Jan/Jun. 2018 hemisphere in stroke p. $13-20$

Valéria Prestes Azolin. valeria_azolin@hotmail.com Fisioterapeuta. Pós-graduada em Reabilitação Físico Motora da Universidade Federal de Santa Maria (UFSM).

Ana Lúcia Cervi Prado. a.lucia@terra.com.br

Fisioterapeuta. Mestra em Desenvolvimento Regional pela Universidade de Santa Cruz do Sul (Unisc). Doutora em Ciências da Saúde pela Universidade de Brasília (UnB). Atua como docente do Curso de Fisioterapia da Universidade Federal de Santa Maria (UFSM).

\section{Resumo}

Contextualização: O Acidente Vascular Cerebral (AVC) é uma perturbação neurológica que ocasiona assimetria na distribuição de peso entre os hemicorpos e consequente dificuldade no controle postural. As diferenças funcionais entre os hemisférios cerebrais originam características distintas neste controle postural, e isso deve ser observado nos tratamentos fisioterapêuticos para melhorar esse déficit. Objetivo: Relacionar o controle postural com o hemisfério acometido no AVC. Métodos: Pesquisa descritiva, transversal do tipo estudo de caso com participantes de um grupo de atenção a hemiplégicos. Foram utilizados: Ficha de Registro dos Pacientes, Escala de Equilíbrio de Berg (EEB), Escala de Deficiência de Tronco (EDT) e Timed Up and Go (TUG). Os dados de caracterização da amostra e as variáveis provenientes dos testes de controle postural foram analisados por meio de estatística descritiva. Resultados: Foram incluídos oito sujeitos participantes do grupo. Embora os resultados não permitam inferir diferença estatisticamente significativa, parece haver evidências de que sujeitos com HCD afetado apresentam maior dificuldade no controle postural em relação a sujeitos com lesão no HCE. Conclusões: No presente estudo, indivíduos com lesão no HCD tiveram escores maiores que os HCE, isso sugere que esses indivíduos têm maiores déficits de controle postural, o que repercute sobre as suas atividades de vida diária e, também, na escolha de um tratamento fisioterapêutico adequado.

\section{Palavras-chave}

Acidente Vascular Cerebral; Controle Postural; Hemisfério Cerebral.

\section{Abstract}

Background: The stroke is a neurological disorder causes asymmetry in weight distribution between hemibodies and consequent difficulty in postural control. The functional differences between the cerebral hemispheres originate distinct characteristic in this postural control and this should be considered in the physical therapy treatments to improve this deficit. Objective: To establish relationship between the postural control and the impaired hemisphere in stroke. Methods: Descriptive study, cross a case, with participants from a group of attention to hemiplegic. They were used: Patient's Registration Form, Berg

\section{Fisiß̊nectus}


Balance Scale (BBS), Trunk Disability Scale (TDS) and Timed Up and Go (TUG). The sample characterization data and the variables analyzed in postural control tests were treated using descriptive statistics. Results: Were included 8 subjects participating in the group. Although the results does not allow infer statistically significant difference, it seems there is evidence that individuals with affected HCD, have greater difficulty in postural control in relation to subjects with injury in HCE. Conclusions: In the present study, subjects with injury in HCD had higher scores than the ones with the HCE affected. This suggests that these individuals have higher deficits in postural control, reflecting on their activities of daily living and on the adoption of a suitable physical therapy treatment.

\section{Keywords}

Stroke; Postural Control; Cerebral Hemisphere.

\section{Introdução}

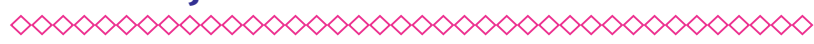

Em escala mundial, o acidente vascular cerebral (AVC) é a segunda principal causa de morte, com aproximadamente 5,7 milhões de casos por ano. É uma doença que ocorre predominantemente em adultos de meia-idade e idosos ${ }^{1}$. Nos últimos anos, o Brasil vem mudando o seu perfil de morbimortalidade, mas o AVC continua sendo a primeira causa de morte e incapacidade no país, gerando deficiências parciais ou completas ${ }^{2,3}$.

O AVC causa incapacidades e leva a alterações funcionais pela deficiência neurológica primária, como déficit de controle postural e dificuldade para realizar as atividades de vida diária. A recuperação da função ocorre em cerca de $50 \%$ a $70 \%$ dos casos de AVC, após seis meses, $50 \%$ dos acometidos ficam hemiplégicos ou hemiparéti$\cos ^{4}$. A hemiplegia é definida como uma sequela neurológica caracterizada por paralisia em um hemicorpo (uma das metades do corpo) devido à doença neurovascular. Ela pode resultar em consequências e comprometimentos em vários níveis de acordo com a área atingida, levando a limitações funcionais e incapacidades contralaterais ao hemisfério lesado, causando alterações de tônus, coordenação e controle postural ${ }^{5}$. Já a hemiparesia é a tendência em manter-se em uma posição de assimetria postural, com distribuição de peso menor sobre o lado afetado, e consequentemente transferência de peso corporal para o lado oposto. Essa assimetria e a dificuldade em suportar o peso no lado afetado interferem na capacidade de manter o controle postural, impedindo a orientação e estabilidade para realizar movimentos com o tronco e membros ${ }^{6}$.

O AVC é considerado uma síndrome com desenvolvimento rápido de sinais clínicos de perturbação focal ou global da função cerebral, com possivel origem vascular e com mais de 24 horas de duração ${ }^{7,6}$. Segundo Bobath ${ }^{8}$, entre as principais alterações geradas por essa doença, estão as alterações na função motora como distúrbios do tônus, presença de reações associadas, perda do mecanismo de controle postural.

0 controle postural tem dois objetivos principais, a orientação e o equilíbrio postural. A orientação postural está ligada ao posicionamento e ao alinhamento do corpo em relação aos outros e também em relação ao ambiente. Já o equilíbrio é o estado em que todas as forças que atuam sobre o corpo estão balanceadas para manter o corpo na posição e orientação desejada. Esse equilíbrio é considerado estável quando o centro de massa é mantido sobre a sua base de apoio ${ }^{9}$.

No AVC o hemisfério acometido, sendo o direito ou esquerdo, tem papéis distintos e que influenciam muito na evolução do caso. Pacientes com lesão no hemisfério cerebral esquerdo (HCE) têm dificuldade para planejar funções motoras; já pacientes com o hemisfério cerebral direito (HCD) acometido têm dificuldade no alinhamento postural e equilíbrio ${ }^{10}$. Saber o lado da lesão e o tipo de comprometimento é extremamente importante para buscar a melhor terapêutica e melhor evolução do caso de cada paciente.

Dessa forma, o presente estudo buscou avaliar o controle postural e sua relação com o hemisfério 
acometido em participantes do Programa Interdisciplinar de Atenção a Hemiplégicos: uma abordagem de terapia em grupo.

\section{Metodologia}

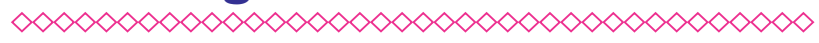

Esta pesquisa caracteriza-se como descritiva, transversal do tipo estudo de caso com abordagem quali-quantitativa. As coletas foram realizadas no Ambulatório de Fisioterapia do Hospital Universitário de Santa Maria (HUSM), durante o período de março a junho de 2016, mediante aprovação prévia pelo Comitê de Ética e Pesquisa da Universidade Federal de Santa Maria (CAAE: 52822416.8.0000.5346, de 22 de março de 2016), sob o número de parecer 1.461 .868 , conforme a Resolução 466/2012 do Conselho Nacional de Saúde sobre pesquisa envolvendo seres humanos.

Foram incluídos no estudo pacientes do Programa Interdisciplinar de Atenção a Hemiplégicos: uma abordagem de terapia em grupo, equiparados pela idade e sexo, que aceitaram participar da pesquisa, assinaram o Termo de Consentimento Livre e Esclarecido (TCLE), foram excluídos do estudo pacientes que tinham comprometimento do nível de consciência, déficit cognitivo, déficit visual, presença de deformidades severas em membros inferiores, não apresentavam ortostatismo de forma independente e que não completaram as avaliações.

A amostra foi do tipo intencional e não probabilística, realizada com pacientes que frequentavam o grupo de hemiplégicos, em que a grande maioria dos participantes tinham acometimento do HCD. Então, primeiramente foram selecionados todos os indivíduos com o HCE acometido $(n=4)$ e comparados com o mesmo número de indivíduos com lesão no HCD e características semelhantes, totalizando oito participantes.

Os procedimentos foram divididos em etapas, sendo a primeira delas o contato com os participantes do grupo, ocasião em que foram explicados todos os processos da pesquisa eaqueles que aceitaram participar voluntariamente assinaram o TCLE.

Posteriormente, foi marcado o dia para a avaliação de cada paciente. Foram avaliados apenas uma vez, sem intervalos, por duas avaliadoras previamente treinadas para aplicar cada uma das escalas.

A primeira etapa da avaliação constituiu no preenchimento da ficha de registro dos participantes, com os dados pessoais e sociodemográficos, como: nome, idade, tempo de AVC, tempo de tratamento, tipo de AVC e lado acometido.

Depois, foi aplicada a Escala de Equilíbrio de Berg (EEB), Escala de Deficiência de Tronco (EDT), e Timed Up and Go (TUG), sendo que previamente as avaliadoras explicaram cada item. A EEB avaliou 0 equilíbrio do indivíduo em situações representativas de atividades diárias, como: ficar em pé, levantar-se, andar, inclinar-se para a frente, transferir-se, virar-se, entre outras. A cada uma das tarefas, podem ser atribuídos valores de 0 (incapaz de realizar) a 4 pontos (realiza com independência). 0 teste avalia tanto a forma como é realizada cada tarefa como o tempo para realizá-la. Os escores totais variam de 0 a 56 pontos, sendo que a máxima pontuação corresponde ao melhor desempenho. Também tem a capacidade a avaliar quantitativamente, podendo ser monitorado o progresso da intervenção terapêutica ${ }^{11,12}$.

Já a EDT avaliou o comprometimento de tronco na hemiplegia em posição sentada, consistindo de subescalas: equilíbrio estático, equilíbrio dinâmico e coordenação, as quais mensuram a qualidade dos movimentos de tronco, apropriado encurtamento ou alongamento muscular e possiveis estratégias compensatórias. Os pacientes têm três tentativas para realizarem cada tarefa. As pontuações máximas das subescalas equilíbrio estático dinâmico e coordenação são 7, 10 e 6, respectivamente. A pontuação total da EDT varia de 0 (pior função de tronco) a 23 (melhor função de tronco) $)^{13,14}$.

Enfım, para fazer a avaliação TUG utilizou-se uma cadeira sem suporte para os braços, cronômetro e um sinalizador para indicar a distância de 3 metros do local onde se encontrava a cadeira. 0 participante foi solicitado a levantar-se da cadeira, deambular 3 metros, regressar e tornar a sentar-se, enquanto o tempo foi cronometrado. Esse teste avalia: equilíbrio estático, transferências de sentado para a posição de pé, estabilidade na deambulação e mudanças no decorrer da marcha ${ }^{15}$. 
Os dados de caracterização da amostra e as variáveis analisadas nos testes de controle postural foram tratados por meio de estatística descritiva (média e desvio padrão) usando o software Excel 2013 (Microsoft Corp.).

\section{Resultados}

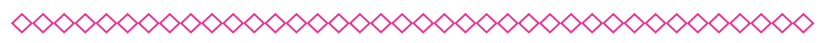

Os dados de caracterização dos oito participantes avaliados, como idade, sexo e histórico do AVC, estão apresentados na Tabela 1.

$\mathrm{Na}$ Tabela 2, estão apresentados os resultados dos testes de controle postural. Apesar de não ter sido possível realizar estatística inferencial, pelos valores médios nota-se que os participantes com o hemisfério cerebral direito acometido tiveram um desempenho mais baixo nos testes aplicados.

\section{Discussão}

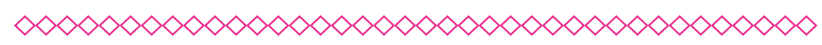

As diferenças funcionais dos hemisférios cerebrais determinam funcionalidades distintas. Ao ocorrer à lesão de algum dos hemisférios cerebrais após o AVC, esperam-se déficits diferentes sobre 0 controle postural, o que, portanto, compromete o comportamento corporal ${ }^{7}$. Este estudo teve como objetivo avaliar o controle postural e a relação com o hemisfério acometido no AVC em participantes do Programa Interdisciplinar de Atenção a Hemiplégicos: uma abordagem de terapia em grupo. Apesar de os resultados inferir diferença estatisticamente significativa e do número reduzido de indivíduos avaliados, parecem existir evidências de que sujeitos com HCD afetado, apresentam maior dificuldade no controle postural em relação a sujeitos com lesão no HCE, principalmente em tarefas que exijam equilíbrio dinâmico, como mostram os escores finais.

Em relação às características da amostra, verificou-se que os indivíduos eram $50 \%$ do sexo feminino, com média de idade de 61,9 anos, variando de 53 a 71 anos. Esses achados vêm de encontro a um estudo ${ }^{16}$ que caracterizou uma amostra de hemiplégicos em relação as suas características clínico-demográficas. Quanto ao tipo de AVC mais frequente, os dados demonstram o mesmo que em outras pesquisas: maior prevalência de AVC do tipo isquêmico $0^{17,18}$.

Outro dado interessante observado em nosso estudo relaciona-se ao tempo que esses pacientes participam das atividades e do tratamento em grupo. A maioria dos indivíduos com lesão em HCD participa há mais tempo do que os indivíduos com lesão esquerda. Mesmo assim, os escores demonstram que possuem maior dificuldade na realização de tarefas que exijam controle postural, o que mais uma vez mostra que o HCD tem relação com o déficit de controle postural. Esse dado corrobora com os encontrados em outra pesquisa que analisou a fisioterapia no tratamento do controle de tronco e equilíbrio pós $A V C$, em que mesmo com terapia semelhante, os indivíduos com lesão no lado direito evoluíram menos ${ }^{19}$.

A busca por métodos avaliativos eficazes é constante, pois, quanto melhor a avaliação, melhor será o resultado de um tratamento e das terapias em grupo. A EEB é uma das escalas mais utilizadas nas pesquisas, por reproduzir diversas atividades comuns de vida diária. Já a EDT, embora não seja muito conhecida, foi escolhida visto que engloba todas as amplitudes de movimento de tronco, a função estática, o equilíbrio dinâmico e a coordenação. Além disso, na EDT a ordem das tarefas ocorre com a dificuldade progressiva, obedecendo à hierarquia da aquisição motora do tronco $0^{9,14}$.

Os resultados dos testes do nosso estudo demonstram que há déficit de controle postural em sujeitos com lesão em HCD. A literatura relata que indivíduos com lesão direita apresentam maior prevalência de déficit postural quando comparados àqueles com acometimento em HCE, apresentando menor equilíbrio postural sentados e em pé20,21.

No que diz respeito à avaliação pela EEB, a média do nosso estudo foi de 40 pontos, evidenciando a diminuição do equilíbrio dinâmico e estático. Estes resultados estão de acordo com a literatura, uma vez que um estudo ${ }^{4}$ aponta uma maior probabilidade de queda e dificuldade no controle postural em indivíduos com pontuação menor que 45 pontos na EEB ${ }^{22}$.

Um bom controle de tronco é caracterizado pela capacidade de a musculatura permitir que o corpo se mantenha na posição vertical, ajustando 
deslocamentos de peso e realizando movimentos seletivos do tronco, mantendo a base de suporte durante movimentos estáticos e dinâmicos, e, assim, proporcionando uma base estável para as funções de controle postural, quando há uma maior dificuldade de manter o controle do tronco, há um maior prejuízo no controle postura|23,24. Isso foi o observado nessa pesquisa, pois os participantes com lesão no HCD tiveram maior dificuldade em realizar as tarefas da EDT, provavelmente pela dificuldade de manter o controle de tronco.

Além disso, nos sujeitos com lesão no HCD, nota-se um maior grau de dificuldade em passar da posição de pé para sentado, de forma independen$\mathrm{te}^{10}$. Isso pode ser observado no presente estudo, pela média final do TUG, em que os sujeitos com lesão direita demoraram e tiveram maior dificuldade para realizar a tarefa.

Um estudo ${ }^{25}$ que analisou o controle postural dinâmico de pacientes com hemiparesia e sujeitos saudáveis encontrou distribuição de peso quase simétrica em sujeitos saudáveis e pacientes com AVC esquerdo, enquanto pacientes com AVC direito desviaram para o lado não acometido. Estes achados vêm de encontro com o nosso estudo, em que os sujeitos acometidos com HCD tiveram resultados inferiores nos testes de controle postural dinâmico.

Benvenuti et al. ${ }^{26}$ defendem que, apesar de os sujeitos com hemiplegia direita e esquerda terem um alinhamento postural e coordenação motora idênticos, observa-se um aumento da oscilação na direção médio-lateral e assimetria postural em sujeitos com hemiplegia esquerda. Esse fato pode ser explicado pelas alterações que possuem na referência interna de estabilidade, derivada de anomalias visuo-espaciais ${ }^{27}$.

Sabendo de todos os déficits de controle postural que sujeitos pós AVC apresentam e que a maioria do grupo estudado é composta por participantes com lesão no HCD, é importante promover atividades que contribuam para diminuir esse déficit. 0 tempo de lesão foi avaliado no estudo, considerando que a fisioterapia precoce produz resultados que dependem da eficácia da intervenção e a capacidade de resposta individual de cada paciente ${ }^{28}$
O'Sullivan $^{5}$ e Alves $^{29}$ relatam que é essencial que o fisioterapeuta seja capaz de reconhecer quando um paciente está sofrendo de algum tipo de disfunção perceptiva, sendo necessário um tratamento adequado para a independência desejada, pois, se for realizada de forma incorreta, o paciente não evolui pelo fato do profissional não ter conhecimento da clínica e, consequentemente, das medidas terapêuticas adequadas. Sendo assim, saber, ter clareza das diferenças funcionais de uma lesão em hemisfério direito e esquerdo, muda a forma de planejar um tratamento, uma reabilitação e das dinâmicas abordadas em grupo.

\section{Conclusão}

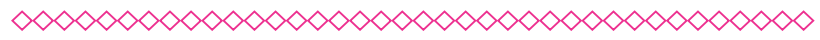

No presente estudo, indivíduos com lesão no HCD apresentaram menores escores que os sujeitos com lesão em HCE, o que sugere que esses indivíduos têm maiores déficits de controle postural. Esse fato repercute diretamente sobre as AVD's, qualidade de vida, independência e bem-estar dos pacientes, por isso, é importante fazer um tratamento fisioterapêutico adequado.

Apesar de os dados do presente estudo não poderem ser generalizados para toda população, são de extrema importância para o grupo estudado, ainda mais se considerarmos que a maioria dos participantes apresentam a lesão justamente no HCD.

\section{Referências}

$\infty \times \infty \times \infty \times \infty \times \infty \times \infty \times \infty \times \infty \times \infty \times \infty \times \infty \times \infty)$

1. Almeida SRM. Análise epidemiológica do Acidente Vascular Cerebral no Brasil. Rev. neurociênc.(Impr.). 2012; 20(4): 481-482.

2. Cavaco NS. Instrumentos de avaliação da função de membros superiores após acidente vascular encefálico: uma revisão sistemática. Fisioter. Pesqui. 2010; 17(2): 178-83.

\section{Santos GM, Souza ACS, Virtuoso JF, Tavares} GMS, Mazo GZ. Valores preditivos para o risco de queda em idosos praticantes e não praticantes de atividade física por meio do uso da Escala de 
Equilíbrio de Berg. Rev Bras Fisioter. 2011; 15(2): 95-101.

4. Woellner, SS; Araujo AGS; Cabral FMH; Uessler PNP; Soares AV. Testes de equilíbrio em pacientes hemiparéticos por AVC. Rev. neurociênc. 2015; 11(1): 32-39.

5. O'sullivan SB, Scmhmitz, TJ. Fisioterapia: avaliação e tratamento. $4^{\mathrm{a}}$ ed. São Paulo: Manole; 2001.

6. Cesário CMM, Penasso P, Oliveira APR. Impacto da disfunção motora na qualidade de vida em pacientes com Acidente Vascular Encefálico. Rev. neurociênc. 2006; 14(1):6-9.

7. Pompeu SMAA, Pompeu JS, Rosa M, Silva MR. Correlação entre função motora, equilíbrio e força respiratória pós Acidente Vascular Cerebral. Rev. neurociênc. 2011; 19(4):614-620.

8. Bobath, B. Hemiplegia em Adultos. $3^{a}$ ed. São Paulo: Manole, 2001; 209p.

9. Meneghetti CHZ, Delgado GM, Pinto FD, Canonici AP, Gaino MRC. Equilíbrio em indivíduos com Acidente Vascular Encefálico: Clínica Escola de Fisioterapia da Uniararas. Rev. neurociênc. 2001; 17(1): 14-18.

10. Pedebos BM, Porto LB, Coppetti F, Balk RS. Avaliação do controle postural e sua relação com o hemisfério acometido em pacientes com acidente vascular cerebral praticando equoterapia. Fisioter. Bras. 2014; 15(1): 22-28.

11. Berg KO, Wood-Dauphinee SL, Williams JI, Gayton D. Measuring balance in the elderly: preliminary development of na instrument. Physiother Can; 1999; 41(6):304-11.

12. Miyamoto ST. Natour J. Brazilian version of the Berg balance scale. Braz J Med Biol Res. 2004; 37(9):1411-21.

13. Verheyden G, Nieuwboer A, Mertin J, Preger $\mathrm{R}$, Kiekens $\mathrm{C}$, Weerdt $\mathrm{W}$. The trunk impairment scale: a new tool to measure motor impairment of the trunk after stroke. Clin Rehabil. 2004; 18(3): 326-433.

14. Castelassi CS, Ribeiro AF, Fonseca VC, Beinotti F, Oberg TD, Lima NMFV. Confiabilidade da versao brasileira da escala de deficiencias de tronco em hemipareticos. Fisioter. Movimento. 2009; 22(2):189-99.

15. Podsiadlo D, Richardson S. The Timed "Up and Go": a test of basic functional mobility for frail elderly persons. JAGS; 2002; 39(2):142-8.

16. Almeida EO, Faleiros BE, Martins C, Lemos SMA, Teixeira AL. Características clínicodemográficas dos acidentes vasculares encefálicos de pacientes atendidos no Hospital Público Regional de Betim, MG. Rev Med Minas Gerais; 2011; 21(4): 384-389.

17. Trindade APNT, Barboza MA, Oliveira $F B$, Borges APO. Influência da simetria e transferência de peso nos aspectos motores após Acidente Vacular Cerebral. Rev. Neurociências. 2011; 19(1):61-67.

18. Ward I, Pivko S, Brooks G, Parkin K. Validity of the Stroke Rehabilitation Assessment of Movement Scale in Acute Rehabilitation: A Comparison With the Functional Independence Measure and Stroke Impact Scale-16. PMR. 2011; 3(11):1013-1021.

19. Ferla FL, Grave M, Perico E. Fisioterapia no tratamento do controle de tronco e equilíbrio de pacientes pós AVC. Rev. neurociênc. 2015; 23(2):211-217.

20. Sayes W, Vereeck L, Truijen S, Lafosse C, Wuyts FP, Heyning PV. Randomized controlled trial of truncal exercises early after stroke to improve balance and mobility. Neurorehabilitation and Neural Repair. 2012; 26(3):231-238.

21. Sena CG, Saes MO, Brod M, Neto VEP. Eficácia do Tratamento Fisioterápico para o controle de tronco em indivíduos acometidos pelo Acidente Vascular Encefálico. Rev Inspirar Mov Saude. 2013; 5(6): 16-19.

22. Borges OS, Filho LENM, Mascarenhas CHM. Correlação entre equilíbrio e ambiente domiciliar como risco de quedas em idosos com acidente vascular encefálico. Rev. bras. geriatr. gerontol. 2010; 13(1): 41-50.

23. Karthikbabu S, Nayak A, Vijayakumar K, Misri ZK, Suresh BV, Ganesan S, et al. Comparison of 
physio ball and plinth trunk exercises regimens on trunk control and functional balance in patients with acute stroke: a pilot randomized controlled trial. Clin Rehabil. 2011; 25(8):709-19.

24. Aguiar PT, Rocha TN, Oliveira ES. Escalas de controle de tronco como prognóstico funcionalem pacientes após acidente vascular encefálico. Acta Fisiatr. 2008; 15(93):160-164.

25. Ikai T, Kamikubo T, Nishi M, Miyani S. Dynamic postural control in patients with hemiparesis. Am J Phys Med Rehabil. 2003; 82(6):463-469.

26. Benvenuti F, Mecacci R, Ferrandino L, Landini L, Baccini M, Stanhope SJ. The Influence of Hemispherical Stroke Side on Spatially Oriented Posture. Gait \& Posture. 2000; 11(2):146.
27. Voos MC, Ribeiro do Valle LE. Estudo comparativo entre a relação do hemisfério acometido no acidente vascular encefálico e a evolução funcional em indivíduos destros. Rev Bras Fisioter. 2008; 12(2): 113-120.

28. Nunes S, Pereira C, Silva MG. Evolução funcional de utentes após AVC nos primeiros seis meses após a lesão. EssFisiOnline. 2005; $1(3): 3-20$.

29. Alves PC, Gaspar RRC. Proposta de Avaliação para o paciente com Síndrome da Heminegligência após Doença Vascular Encefálica. (Relato de Caso). Reabilitar. 2002; 4(17): 36-42. 


\section{Anexos}

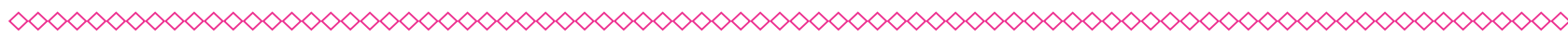

Tabela 1 - Caracterização da amostra

\begin{tabular}{|c|c|c|c|c|c|c|}
\hline & $\begin{array}{l}\text { Idade } \\
\text { (anos) }\end{array}$ & Sexo & $\begin{array}{c}\text { TA } \\
\text { (anos) }\end{array}$ & TT (anos) & $\begin{array}{l}\text { Hemisfério } \\
\text { Acometido }\end{array}$ & Tipo de AVC \\
\hline DF & 65 & $M$ & 14 & 8 & HD & isquêmico \\
\hline OM & 55 & $M$ & 13 & 9 & HD & isquêmico \\
\hline MGP & 66 & $\mathrm{~F}$ & 10 & 4 & HD & isquêmico \\
\hline NT & 58 & $\mathrm{~F}$ & 4 & 4 & HD & isquêmico \\
\hline $\mathrm{IL}$ & 67 & $M$ & 7 & 5 & $\mathrm{HE}$ & isquêmico \\
\hline AM & 60 & $M$ & 13 & 2 & HE & isquêmico \\
\hline MSB & 71 & $F$ & 13 & 9 & HE & hemorrágico \\
\hline \multirow[t]{2}{*}{ MCS } & 53 & $\mathrm{~F}$ & 3 & 2 & $\mathrm{HE}$ & hemorrágico \\
\hline & $61,9 \pm 6,3$ & & $9,1 \pm 4,2$ & $5,4 \pm 2,9$ & & \\
\hline
\end{tabular}

Valores expressos em média DPP; TA= Tempo de AVC; TT= Tempo de tratamento fisioterapêutico.

(clique para voltar ao texto)

Tabela 2 - Resultados dos testes de controle postural, para a amostra estudada

\begin{tabular}{ccc} 
& \multicolumn{2}{c}{ Hemisférios Acometidos } \\
& HCD $(\mathrm{n}=4)$ & HCE $(\mathrm{n}=4)$ \\
TUG (seg) & $27,7 \pm 24,8$ & $18,2 \pm 10,6$ \\
EDT (escore) & $10,5 \pm 2,6$ & $14,7 \pm 2,1$ \\
EEB (escore) & $40 \pm 10,2$ & $48 \pm 8,8$ \\
\hline
\end{tabular}

Valores expressos em média $\pm D P ; T U G=$ Time Up and Go; EDT= Escala de Deficiência de Tronco; EEB= Escala de Equilíbrio de Berg. 